\title{
Zwischen Science Fantasy und ontologischer Politik. Künstlerischer Technomaterialismus jenseits der Geschlechtergrenzen ${ }^{1}$
}

In den 1990er Jahren entwickelte sich Bio-Art, ein Bereich der Kunst, der die Verfahren, Techniken und das Material aus den Laboren der Biound Gentechnologien künstlerisch gestaltet und ästhetisiert. Diese Kunst thematisiert Fragen der Technikentwicklung, der Transformation von Mensch-Technik-Materie-Relationen und erzeugt sin vivo< wie auch sin vitro Möglichkeiten der Konfrontation und der gesellschaftlichen Auseinandersetzung. Ein bekanntes Beispiel ist das Bild eines transgenen Lebewesens, das der Künstler Eduardo Kac im Jahr 2000 in die Welt brachte: ein grün leuchtendes Kaninchen, in dessen Genom ein aus einer Tiefseequalle stammendes Gen zur Herstellung eines fluoreszierenden Protein eingebaut wurde. Kac deklarierte den grünen Hasen als neues Mitglied seiner Familie und löste eine öffentliche Debatte über die ethischen und sozialen Implikationen der Gentechnologie aus (Kac 2000).

Der vorliegende Beitrag interessiert sich dafür, wie in den Projekten und Produkten der Bio-Art ein diskursiver Raum erzeugt wird, in dem die mit den technologischen Entwicklungen verbundenen Erfahrungen der Verflüssigung von gesellschaftlichen Bedeutungen und Sicherheiten die semiotisch-materiellen Zusammenhänge von Mensch, Maschine und Materie betreffend sichtbar und erfahrbar werden. Dabei ist auffällig, dass die feministische Frage nach den mit Hilfe der Bio- und Gentechnologien hervorgebrachten Dynamisierungen von Geschlechterkonstellationen samt kategorialer Verschiebungen zumeist nur abstrahiert in Erscheinung tritt: als Frage danach, wie materielle Grenzen und Bedeutungen von Körpern, Lebewesen und Arten temporär und lokal in Kraft gesetzt werden. Diese Abwesenheit konkreter geschlechtlicher Figurationen und Bedeutungen überrascht, beziehen sich doch viele künstlerische Bearbeitungen technisch-lebendiger Materie explizit auf Donna Haraway, vor al-

1 Ich danke den anonymen Gutachter_innen für die sorgfältige Lektüre und die dezidierten Rückmeldungen. Špela Petrič danke ich für die Genehmigung zum Abdruck der Fotos ihrer Kunstwerke und Miha Turšič dafür, dass er das Phytoteratology-Bild zur Verfügung gestellt hat. 
lem auf ihre Idee, sich den nicht-menschlichen Lebewesen und Dingen "verwandt zu machen" (Haraway 2018 [2016], 137) und dabei neue, nichtanthropozentrische Perspektiven auf die Bandbreite der Formen lebender Materialität einzunehmen. Warum aber, so fragt dieser Beitrag, kommen die künstlerischen Bezugnahmen auf Donna Haraways "spekulativen Feminismus" (Haraway 2018 [2016], 20) zumeist ohne die explizite Auseinandersetzung mit der Kategorie Geschlecht aus und stützen sich stattdessen auf Konzeption und Imagination von Formen und Kooperationen eines anderen, neuen sozialen Miteinanders, in denen die geschlechtliche Differenzierung nicht im Vordergrund steht?

Haraway selbst hat seit der Veröffentlichung ihres Cyborg-Manifesto ihr Arbeiten und Denken als Bestandteil eines feministischen Projekts gegen kategoriale Vereindeutigungen und identitätspolitische Festlegungen verstanden. Als sie 1985 schrieb »Ich wäre lieber eine Cyborg als eine Göttin" (Haraway 1995 [1985], 72) argumentierte sie damit gegen eine essentialistische Vorstellung von Weiblichkeit, Geburt und Mutterschaft, die Natürlichkeit feiert. Vielleicht, so überlegte sie, besteht die entscheidende Herausforderung von Gentechnologie und Reproduktionstechnologien darin, dass sie das Vertrauen in die Naturhaftigkeit des Körpers erschüttern und unsere Vorstellung davon verändern, wo Körper enden und die Umwelt oder andere Menschen beginnen (Haraway 1995 [1989], 174). Die politische Dimension dieser technischen Entwicklung bestand für Haraway vor allem darin, dafür zu sorgen, dass jetzt, da die (vermeintliche) Unberührtheit der Körper und ihrer (scheinbaren) passiven Materialität nun einmal verloren ist, sich auch Frauen die Technologien aneignen und neue Signifikationspraxen, andere Umgangsformen und Arten der Verkörperung entwickeln. Denn sonst seien es nach wie vor andere, die diese Technik nutzen, um über die Körper von Frauen zu herrschen und die neuen, hybriden, biotechnisch gemachten Geschlechtskörper würden ohne die Partizipation von Frauen bzw. Aktivistinnen figuriert (Haraway 1992 sowie Haraway 1995 [1989], 59-61).

Seither arbeitet Haraway an der Ausdifferenzierung eines Figuren-Kabinetts materiell-semiotischer Akteur_innen technowissenschaftlich-kultureller Produktion. Sie sollen dabei helfen, Wissensgenerierungen und Grenzverschiebungen der veränderten historisch spezifischen Bedingungen, die mit den technologischen Entwicklungen verbunden sind, kritisch-produktiv werden zu lassen. In jüngsten Zeit plädiert sie vor allem dafür, den Umwelt zerstörenden Egoismus des Anthropozäns hinter sich $\mathrm{zu}$ lassen und an der Herstellung neuer sozialer Beziehungen mit der Welt und ihren Geschöpfen zu arbeiten (Haraway 2003; 2008; 2018 [2016]). 
Dafür, so ihre Überzeugung, sei es erforderlich, dass die Menschen auf die Lebensumstände von Pflanzen, Tieren und auch die von Bakterien und Korallen so gefühlvoll achten, wie auf das Gedeihen ihrer Kinder. Die Menschen müssten, so die Forderung, "empathisch" gegenüber allen Lebewesen werden. Sie sollten "Tentakel" und "Fühler» entwickeln, mit deren Hilfe sie einem "sensiblen Materialismus" folgend die "Mitwesen» in ihrer Umgebung erspüren und sich ihnen ähnlich und "verwandt" fühlen. Denn: Alle Lebewesen gemeinsam bildeten den "Komposthaufen« des Lebens, den »Humus« des Planeten, (Haraway 2018 [2016], 80). Mit dem Motto "Make kin, not babies" schlägt sie vor, die eigene Fortpflanzung sparsam zu betreiben, wenige menschliche Kinder entsprechend aufzuwerten und darüber hinaus verwandtschaftliche Beziehungen zu Technik und Tieren einzugehen und neue Arten in unsere >Familien` aufzunehmen (Haraway 2018 [2016], 142).

In diesem Sinne verstehen sich auch viele künstlerische Arbeiten zum Thema Bio-Technologien, die in den letzten Jahren im Kontext der BioArt entstanden sind, als Versuche einer eigenständigen und kritischen Aneignung neuer technisch-technologischer Möglichkeiten und Visionen (Rapp/de Luz 2018). Welche Rolle und Bedeutung der Kategorie Geschlecht dabei zukommt, soll in diesem Beitrag anhand eines aktuellen Bio-Art-Projekts und dessen Einbettung in gegenwärtige theoretische und politisch-gesellschaftliche Kontexte und Diskurse betrachtet werden. An den künstlerischen Aktivitäten der slowenischen Mikrobiologin und Bio-Art-Künstlerin Špela Petrič soll exemplarisch gezeigt werden, welche Klassifikationen diese Kunstpraxis strukturieren und wie diese theoretisch und praktisch im sozialen Feld der Bio-Art situiert sind. Dazu ist es zunächst erforderlich, das Bio-Art-Feld gesellschaftlich und diskursiv-theoretisch zu verorten und so werden in einem ersten Schritt die Schnittstellen und Bezüge von Bio-Art und dem sogenannten posthumanistischen Feminismus sowie die Konzepte von Open Science und Citizen Science skizziert, die als Leitlinien und Rahmung der bio-philosophischen Praxis von Bio-Art eine zentrale Rolle spielen. Diese theoretischen Rahmungen stellen der künstlerischen Suche spekulativ-zukünftiger Möglichkeiten von »Art-Genossen(schaft) ( (Haraway 2018 [2016], 24) eine Reihe von Zugängen, Methoden, Bildern und Figuren zur Verfügung, die - das soll dann im zweiten Schritt am Beispiel der Projekte von Špela Petrič gezeigt werden - zwischen Eigensinnigkeit und Affirmation hin und her pendeln. 


\section{Bio-philosophische Begriffsarbeit und die Rolle von Kunst}

Eine Reihe von Autorinnen, die mit dem Label >Posthumanistischer Feminismus versehen werden - z. B. Donna Haraway (1992), Marilyn Strathern (1992), Karen Barad (2003), Elisabeth Grosz (2008), Stacy Alaimo und Susan Hekman (2010), Rosi Braidotti (2014) - stellen die Notwendigkeit heraus, neue Geschichten, neue Methoden und neue Figuren für kritische Analysen der Gegenwart zu entwickeln. In einer Welt, in der die Aktivitäten des globalen Biokapitalismus und techno-biopolitische Entscheidungen zunehmend Bedeutung erlangen, seien Konzepte und Figuren nicht als Werkzeuge zum Begreifen der Welt anzusehen, sondern vielmehr als performative und generative Entitäten, die aktiv an der Re(kon) figurierung der Welt teilhaben. Figuren und Konzepte, so die Überzeugung dieser Denkerinnen, sind materiell und leibhaftig, und sie haben ethische und politische Konsequenzen, die es einerseits theoretisch zu analysieren und andererseits politisch zu bearbeiten gelte. Dementsprechend ist die vielzitierte Figur des "Cyborgs" eben nicht nur eine Beschreibung und Repräsentation bereits existierender Mensch-Maschine-Schnittstellen, sondern ein analytisches Konzept und eine kritische Erzählfigur, die, wie Figuren und Figurationen überhaupt, angesehen werden können als "verdichtete Kartographien anfechtbarer Welten mit der Macht, festgefahrene Identitäten und Gewissheiten aufzusprengen" (Haraway 1997, 17).

Im Prozess der Suche nach Formen, Möglichkeiten, Mitteln und Wegen der Ausgestaltung dieses Dynamisierungspotenzials von Figuren, Narrativen und Konzepten spielen künstlerische Annäherungen und Entwürfe eine wichtige Rolle. Das künstlerische Portfolio taktischer Möglichkeiten zu nutzen, ist zentraler Bestandteil des Haraway'schen Projekts der "wissenschaftlich-künstlerischen Verweltlichungen für das Leben auf einem beschädigten Planeten" (Haraway 2018 [2016], 119). Die konkrete Kunstpraxis aber - und davon abstrahiert Haraway weitgehend - steht ihrerseits auch im Widerstreit zwischen Sammler_innen und Kurator_innen und agiert zwischen der Macht institutioneller Apparate und ihrer Mechanismen, Rankings und Förderprogramme, Zeitschriften und wechselnden Moden. Dieses gesellschaftliche Bedingungsgefüge künstlerischer Arbeit generiert und strukturiert die (Bio-)Kunst, so wie es umgekehrt auch durch diese Arbeit gestaltet und verändert wird.

Im Bereich der Bio-Art sind viele Künstler_innen dem politischen Aufruf zur eigenständigen Aneignung der Technologien son unten 'gefolgt (Kurzweill 2000; Serres 2013; Mêtraux et al. 2016) und haben sich von dieser Aufforderung in ihren künstlerischen Projekten leiten lassen (vgl. 
die Überblicke zum Thema Bio-Art von Reichle 2005; Mitchell 2010; Myers 2015). Darüber hinaus haben sich Praktiken der Aneignung im Rahmen der Do-It-Yourself-Biology etabliert, dem sog. Bio-Hacking als sozialer Bewegung. ${ }^{2}$ Solche Aktivitäten finden mittlerweile im Kontext von Citizen Science (z.B. Eggleson 2014; Vohland/Göbel 2017) auch Eingang in akademische Projekte der Naturwissenschaften und Technikentwicklungen, denen es wichtig ist, die Begleiterscheinungen und möglichen Risiken ihrer Anwendungen partizipativ zu erarbeiten, transparent $\mathrm{zu}$ machen und dazu auch mit den Künsten zu kooperieren. Beispielsweise startete im Oktober 2018 an der Technischen Universität Berlin das zweijährige Citizen Science Projekt "Mind the Fungi« als Kooperation des Instituts für Biotechnologie/ Abteilung Angewandte und Molekulare Mikrobiologie mit dem Art Laboratory Berlin, einem zentralen Akteur der deutschen Bio-Art-Szene. In dem Projekt werden Pilze als nachwachsende pflanzliche Rohstoffe biotechnologisch so bearbeitet hergestellt, dass sie als Verpackungsmaterial und Baustoffe verwendet werden können. Die von der TU Berlin zur Verfügung gestellten Projektkosten betragen 300000 Euro, von denen ein Teil auch für den Bereich der Wissenschaftskommunikation verwendet wird, für den das Art Laboratory Berlin zuständig ist. ${ }^{3}$

Die Zusammenarbeit von Naturwissenschaft, Biotechnologie und Kunst hat nach Bekunden der Beteiligten das Ziel, die blinden Flecken des an wissenschaftlicher Empirie ausufernden Wissensbestands auszuleuchten. So erläutern die Berliner Kurator_innen Regine Rapp und Christian da Luz im Interview, dass es bei dieser Ko-Laboration auch darum gehe, bloße Fortschrittsideologien, wie sie von den Informationstechnologischen Unternehmen im Silicon Valley propagiert werden oder in transund posthumanistischen Utopien von beispielsweise humanoiden Robotern zum Ausdruck kommen, zu differenzieren und ggf. auch Grenzen der Vereinnahmung von Mensch und Maschine aufzuzeigen (vgl. MüllerJung 2018, 3).

Das Projekt »Mind the Fungi« steht im Kontext von übergreifenden Bemühungen, Wissenschaft zu öffnen und Anschlüsse zur Öffentlichkeit und zu sogenannten Laien herzustellen, wie zum Beispiel das mit Mitteln des EU-Programms Horizon 2020 - Science with and for Society (SWAFS) geförderte Projekt ORION - Open Science I Horizon2020, in dem sich

\footnotetext{
Vgl. z.B. https://www.hackteria.org/.

https://www.forschung.tu-berlin.de/servicebereich/menue/forschung_an_der_tu/ citizen_science_projekte_2018/mind_the_fungi/.
} 
neun führende europäische Forschungsinstitute, nationale Fördereinrichtungen, Unternehmen und zivilgesellschaftliche Organisationen zusammengeschlossen haben, um soziale Partizipation und Transparenz in der Forschung durch "Co-Creation", durch Experimente, Benchmarking und Training zu fördern und um institutionelle Strukturen, Kultur und Verhalten in Richtung Open Science zu verändern. ${ }^{4}$ Im Zuge solcher Öffnungsbestrebungen wird auch der Integration von Kunst in die Wissenschaften ein Platz im Rahmen der Forschungsförderung zugewiesen. Zu diesem Zwecke werden neue Veranstaltungsformate entwickelt und Einrichtungen gegründet, die Wissenschaft, Kunst und Innovation zusammenführen (sollen) - ein Beispiel dafür ist das Festival STATE, das im Oktober 2018 in Berlin eine "Kunst- und Wissenschaftsgalerie und -agentur» eröffnete. ${ }^{5}$ Und auch die Einbeziehung von "artistic research" (Haarmann 2019) in Forschungsprojekte hat die Auseinandersetzung um die methodologische Bedeutung von Kunst in der Wissenschaft intensiviert. Dabei ist die These relevant, dass durch die Arbeit mit dem Material und durch das Material neue Problemlösungen und Darstellungsformen hervorgebracht würden, dass also Objekte wie Akteur_innen gleichermaßen zur Konstruktion von Ergebnissen beitragen. Zudem wird angenommen, dass die Beteiligung von Kunst Forschungsprojekte ergänze und bereichere, indem sie das den künstlerischen Praktiken inhärente sensuell-implizite Wissen sowie Prozesse von Erkenntnis veränderten und zur Produktion neuen Wissens beitrügen (Lehner 2017).

Wie sich im Zuge dessen die künstlerischen Aktivitäten zwischen InDienstnahme und Affirmation einerseits und Differenzierungspotenzial und Kritik andererseits positionieren und entwickeln werden, dürfte von Projekt zu Projekt unterschiedlich verlaufen und wartet noch auf bilanzierende Analysen dieser neuen Funktion und Aufgabe. Auf jeden Fall lässt sich konstatieren, dass die Ko-Laboration von Kunst, Wissenschaft, Wissenschaftspolitik und Privatwirtschaft im biotechnologischen Labor sowie als wissenspolitisches Laboratorium jetzt auch in die deutsche Forschungslandschaft Einzug erhalten hat.

4 https://ec.europa.eu/programmes/horizon2020/en/h2020-section/science-and-society.

5 https://state-studio.com. 
Bislang gab es Kooperationen von Kunst, Natur- und Technikwissenschaften vor allem in Australien ${ }^{6}$ und Nordamerika ${ }^{7}$, wo Bio-Art auch in der Form von Studiengängen organisiert ist. In Europa bildet Ljubljana ein bedeutendes Zentrum der Bio-Art; hier arbeiten Künstler_innen auf dem Campus der Biowissenschaften. ${ }^{8}$ Die dortigen Verbindungen von Biowissenschaft und Kunst haben zum einen das Ziel, Forschungen sichtbar zu machen und sie für Interpretationen von Nicht-Wissenschaftler_innen zugänglich zu machen. ${ }^{9}$ Zum anderen geht es bei dieser institutionalisierten Ko-Laboration auch darum, mittels der künstlerischen Artikulation von Prozessen der technischen Bearbeitung des Lebendigen eine gesellschaftliche Verständigung über das Leben und das soziale Miteinander in Gegenwart und Zukunft zu initiieren. ${ }^{10}$

Diese offiziell formulierten Anliegen sind anschlussfähig an die Überlegungen Donna Haraways, da sie über das konkrete naturwissenschaftlich-technische Ansinnen hinaus - das beispielsweise darin besteht, Bakterien zu kultivieren, die sich von Plastik ernähren, oder darin, Zellen aufzubewahren bzw. zu züchten, deren körperlicher Ursprungsort verloren gegangen ist - auch dazu angelegt sein können, um es mit Donna Haraway zu sagen: "Responsabilität« für starke und gefährdete Orte und Wesen zu kultivieren (Haraway 2018 [2016], 143; 159). Kunst, so hoffen sowohl die Bio-Art-Protagonist_innen als auch Haraway, biete die Möglichkeit, über die gängigen Zukunftsbilder hinaus solche materiell-semiotischen Verbindungspraktiken zwischen Gegenwart und Zukunft zu entwerfen und zu erproben, die das Bestehende $z u$ transzendieren vermögen und Neues vorstellbar werden lassen.

In Haraways eigener Sprache, die als Ausdruck der Suche nach neuen, eigenwilligen, nicht kontaminierten Konzepten und Figuren stetig neue Begriffe zu entwickeln und einzuführen bemüht ist, ist dabei die Rede von "wissenschaftlich-künstlerischen Verweltlichungen", »in denen WissenschaftlerInnen, KünstlerInnen, ganz normale Mitglieder von Gemeinschaften und nicht-menschliche Wesen einander in den jeweiligen Pro-

6 z.B. das Labor SymbioticA an der University of Western Australia in Perth. http:// www.symbiotica.uwa.edu.au.

7 z. B. das SVA Art and Science Laboratory an der School of Visual Arts in New York. https://bioart.sva.edu.

8 Orte von Bedeutung sind auch die Ars Electronica in Linz, Österreich (https://ars.elec tronica.art), die Organisation Waag-Technology and Society in Amsterdam/Niederlande (https://waag.org/) oder auch die Bio-art-Society im finnischen Kilpisjärvi (https:// bioartsociety.fi).

9 Vgl. www.biotehna.com.

10 https://assets.openscience-files.or.at/_/cc/a0/cca0584f.pdf. 
jekten und Lebensvollzügen umfassen; mit der Zeit brauchen sie einander auf vielfältige, leidenschaftliche, körperliche und bedeutungsvolle Art und Weise. Jedes Projekt ist ermutigend in tödlichen Zeiten. Sie alle sind sympoietisch, symbiogenetisch und symanimaginativ« (Haraway 2018 [2016], 102). Bezogen auf die bio-künstlerische Ko-Laboration zwischen Künstler_innen, Labor, Material und Publikum legt Haraways Perspektive die Frage nahe, wie Bio-Art-Projekte Kategorien des Körperlich-Lebendigen in Bewegung bringen und wie dabei Geschlecht als eine Dimension des »Vielfältige(n), Leidenschaftliche(n), Körperliche(n) und Bedeutungsvolle(n)« (ebd.) künstlerisch zum Ausdruck gebracht wird. Dies soll im Folgenden anhand eines ausgewählten Beispiels - den Arbeiten Strange Encounters with Vegetal Others und Phytoteratology der promovierten Biochemikerin und Künstlerin Špela Petrič - betrachtet werden.

\section{Verhandlungen des Vegetativen - Ein Ausstellungsprojekt}

Špela Petrič verbindet in ihrer künstlerischen Praxis Naturwissenschaften, neue Medien und Performance, indem sie laborwissenschaftliche Methoden in verschiedene kulturelle Kontexte transferiert und lebende Systeme in Verbindung mit unbelebten Systemen erzeugt, die lebensechte Eigenschaften aufweisen. Sie bezeichnet ihre künstlerischen Experimente, die sie auch in Kunst- und Wissenschaftsworkshops vorstellt, als »TerRabiology « - eine relational orientierte ontologische Sicht auf die Entwicklungen und Formationsprozesse auf der Erde, die anthropologische, psychologische und philosophische Fragen nach dem Prozess des Lebens aufwirft und einen kritischen Diskurs zwischen Labor, Kunst und Öffentlichkeit erzeugen will. ${ }^{11}$

Im Herbst 2018 hat Petrič im Art Laboratory Berlin ihr dreiteiliges Ausstellungsprojekt "Strange Encounters with Vegetal Others" präsentiert, in dem sie die Begegnung mit Pflanzen als >dem Anderen zum Thema macht. Petrič nimmt die Ko-Laboration zwischen den beiden Spezies 'Mensch und ’Pflanze in den Blick. Da Menschen zum Überleben auf Pflanzen angewiesen sind, thematisiert die Künstlerin Methoden und Praktiken der Kultivierung und der Pflege sowie Frage nach den Unterschieden und Zusammenhängen zwischen der Biologie der Pflanzen und der humanen Ontologie: einerseits eher untätig, vegetativ im grundle-

11 https://www.spelapetric.org/\#/strange-encounters; letzter Zugriff am 6.6.2019. 
genden Sinn, andererseits, wie es im Flyer zur Ausstellung heißt: "ausgestattet mit vielen noch unerforschten Formen der Intelligenz. Doch die Wissenschaft offenbart eine komplizierte Welt schwer nachvollziehbarer chemischer Konversationen, zwischenartlicher Netzwerke und nicht zentralisierter Operationen, die unserer eigenen Existenz fremd sind. Mit ihrer Arbeit schlägt Petrič neuartige Formen der Kommunikation zwischen Mensch und Pflanzen, gegenseitiger Erkenntnisse und Ansätze eines möglichen Austauschs vor" (Art Laboratory Berlin 2018). Auch wenn man dem hier verwendeten niedrigschwelligen Begriff von Kommunikation nicht folgen mag, Petričs Kunst stellt die Frage nach der sozialen und der informationellen Verschränkungen von Perspektiven, konkret: die zwischen Pflanzen und Menschen.

Es ist ein Charakteristikum von Bio-Art, dass diese Kunst in für das menschliche Auge unsichtbare Bereiche vordringt und dabei auch die Techniken der Sichtbarmachung und deren Emergenz-Potenzial zum Thema macht. Aus konstruktivistischer Perspektive ist auch die Visualisierung von Mikroorganismen ein Vorgang ihrer Erzeugung, der im Prozess der Herstellung manipuliert und gestaltet werden kann, und diese performative Dimension ist Element der Laborforschung wie auch der Kunst. Genau diesen doppelten Prozess der Hervorbringung von Vegetativem sichtbar zu machen, ist Thema von Petrič im zweiten Teil ihrer Ausstellung, im Projekt »Strange Encounters: Metaphysics, Algae and Carcinoma", eine Dokumentation der "Mikroperformance" von Algen und menschlichen Krebszellen aus dem Jahr 2017. Beobachtet durch eine Mikroskopkamera erlebt die Zuschauer_in, wie sich Zellen eines Blasenkarzinoms und die einzellige Chlorella-Alge in einem Zellkulturglas in vitro begegnen, wie sie sich - mikrobiologisch gesprochen - Raum aneignen, und wie es im Zuge dessen dazu kommt, dass die menschlichen Zellen die Mikroalgen verzehren. Dieses Phänomen, das die Biochemikerin Petrič nicht antizipiert hatte, wirft die Frage auf, was wir hier eigentlich beobachten können: die Macht bzw. Dominanz der menschlichen Biologie oder vielmehr die Macht eines künstlerischen Experiments und seine biopolitische Instrumentierung? 


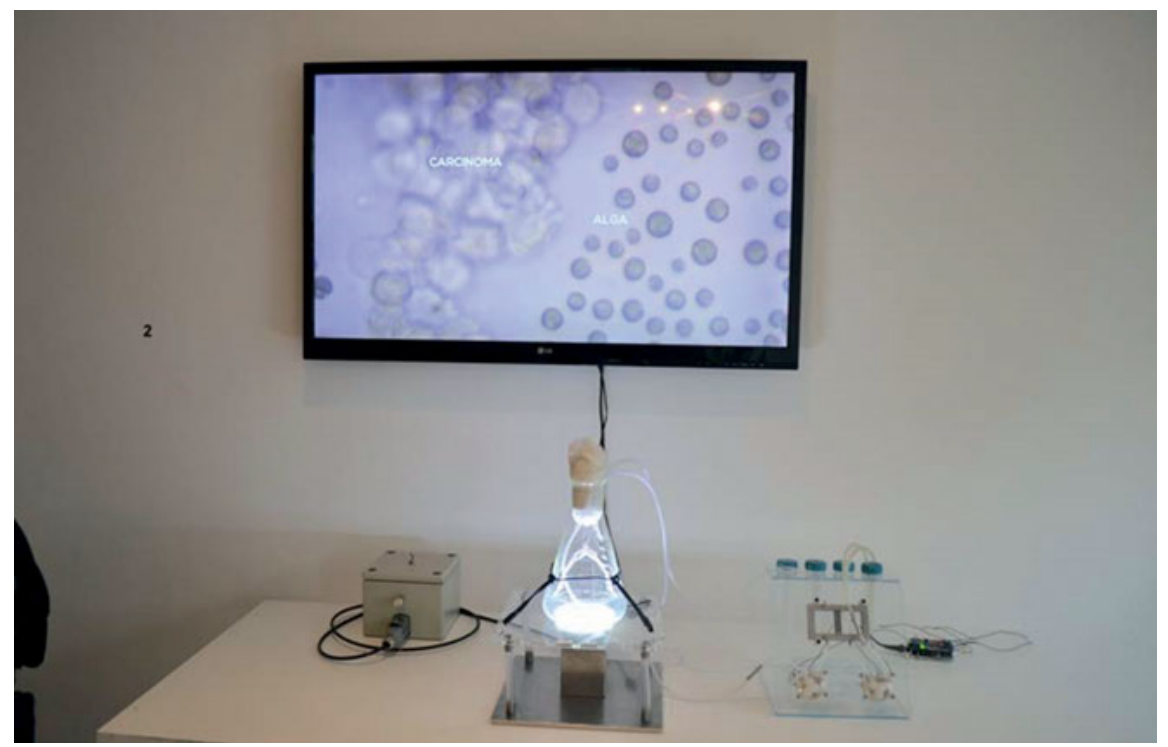

Spela Petrič: Strange Encounters: Metaphysics, Algae and Carcinoma, 2017, (C) Katharina Liebsch

Den ersten Teil der Ausstellung bildet die Arbeit "The Plant Sex Consultancy«, die Petrič zusammen mit Pei Ying Lin, Dimitris Stamatis und Jasmina Weiss 2014 produziert hat. Hier entwerfen die Künstler_innen designbasierte Lösungen zur Bestäubung von sechs Pflanzenarten, die sowohl Elemente von Medizinprodukten als auch von Sexspielzeug aufnehmen. Die präsentierten Techniken spielen mit der Ästhetik von Bewässerungsanlagen und blumigem Sexspielzeug und stellen damit zum einen die Frage nach der Empfindungsfähigkeit von Pflanzen. Zum anderen wecken sie Assoziationen zur Zukunft von Bestäubung in einer Welt ohne Insekten und $\mathrm{zu}$ den Möglichkeiten der künstlichen Fortpflanzung und technisch assistierter Reproduktion aller Gattungen und Spezies. 


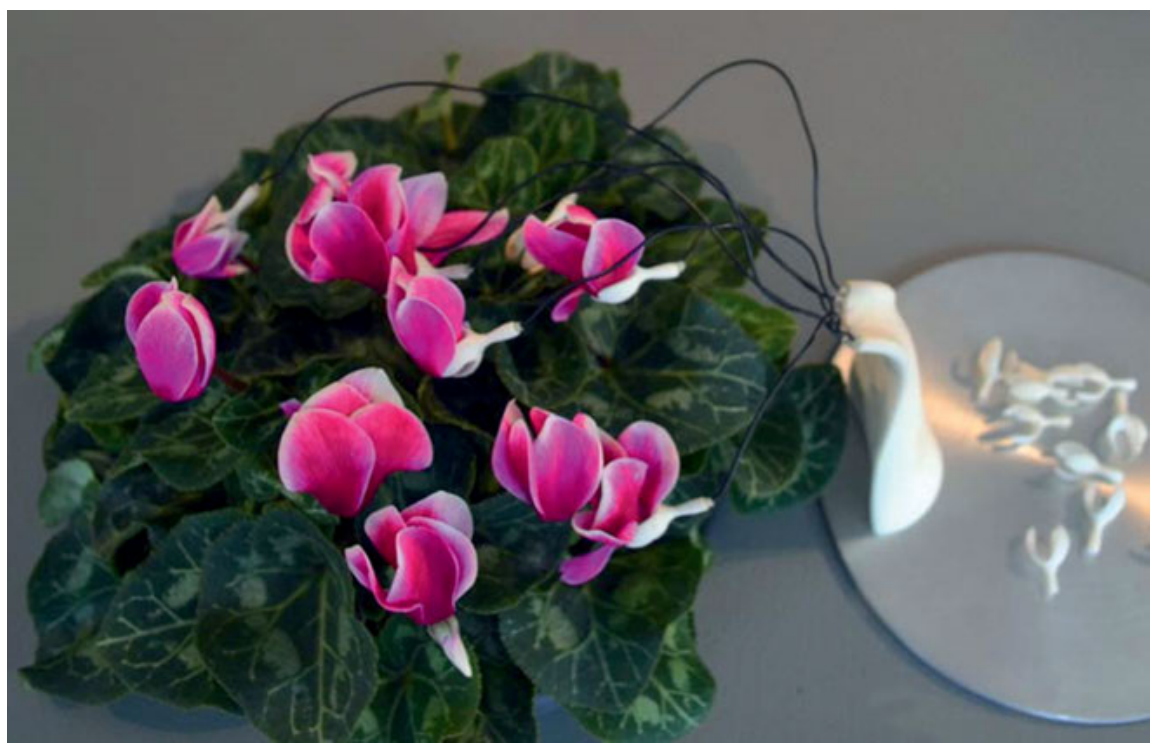

Špela Petrič, Pei Ying Lin, Dimitris Stamatis, Jasmina Weiss: "The Plant Sex Consultancy“, 2014, (C) Katharina Liebsch

Den dritten Teil der Ausstellung bildet die fotografische Dokumentation einer zwanzig-stündigen Ko-Laboration zwischen der Künstlerin und einem Feld von Kresse-Pflanzen, einer Performance mit dem Namen "Skotopoiesis", was ,Von Dunkelheit geformt bedeutet. Hier hatte Petrič fast einen ganzen Tag lang in einem dunklen Raum vor einem exakt ausgeleuchteten Feld von keimenden Kressepflanzen gestanden. Da sie im Licht des Scheinwerfers steht, wirft ihr Körper einen scharf gestochenen Schatten auf das Kresse-Feld. Wenn das Publikum lange genug ausharrte, konnte es erleben, wie der Schatten Petričs die Kresse verblassen ließ und die Keimlinge am Schattenrand sich verlängerten.

Eine Interaktion? Das Zusammenspiel des verkleinerten (Schatten-) Körpers der Künstlerin und der sich vergrößernden Pflanzen thematisiert die Bedeutung von Intervention durch Menschen und Technik. Zugleich zeigt die Langzeit-Performance der Künstlerin und ihr stundenlanges Stillstehen den Zusammenhang von Körperlichkeit und Zeit bzw. Zeitgefühl, der bei verschiedenen Lebewesen jeweils unterschiedlich ist. Mit der Sichtbarmachung der Differenzen zwischen den Eigenzeiten von Entitäten (innerhalb von Phänomenen bzw. Ganzheiten) ist auch die Frage nach der Synchronisation von Zeitordnungen unterschiedlicher Lebewesen verbunden, eine Überlegung, die für den Gedanken der Nachhaltigkeit zentral ist. 


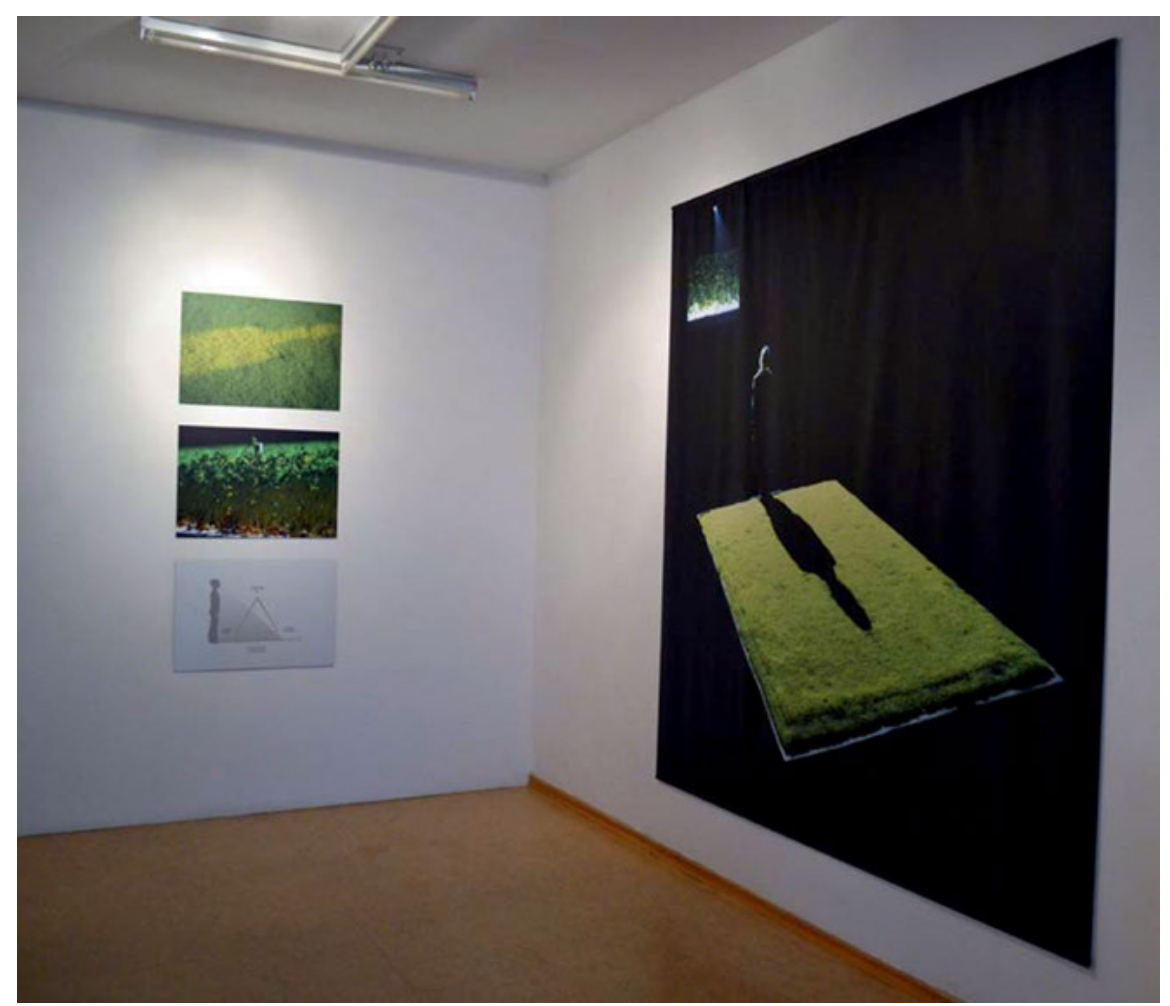

Špela Petrič: "Skotopoiesis« produziert von der Kapelica Gallery, 2016, C Katharina Liebsch

Für eine weitere Arbeit, in der Petrič gleichermaßen darum bemüht ist, die Perspektive von Organismen einzunehmen und an einem System Natur teilzunehmen, das sie mit Hilfe von Methoden der Biotechnik bearbeitet und verändert, erhielt die Künstlerin 2016 den White Aphroid Award des Projekts "Risk Change«, das vom Creative Europe Program of the European Union mitfinanziert wird. In der Serie "Phytoteratology» (also: der Kunde von den pflanzlichen Monstern) schuf sie "Phytopolutanten« - Informationsgemische aus Mensch und Pflanze, genauer: zwischen Zellen der Künstlerin und Kresse. Mit Hilfe biotechnischer Verfahren (re)produzierte sie pflanzenmenschliche Entitäten, indem sie aus pflanzlichem Gewebe »Pflanzenembryos« herstellte, deren Wachstum sie mit Sexualhormonen aus ihrem eigenen Urin förderte und die dann besondere Formen und Ausgestaltungen entwickelten. Petrič interpretiert dies so, dass die Hormone der Künstlerin bei den "Pflanzenembryonen« eine Erinnerung ihrer Anwesenheit erzeugten und diese wiederum als Antwort darauf eine einzigartige Form entwickelten. 
In der gegenwärtigen Zeit vielfältiger politischer, ökologischer und sozialer Umbrüche erzeugt Petrič »kleine grüne Monster« als ausgetretene Wesen, als Neugeborene von angeborener Intra-Aktion (Barad 2007), TransSpezies-Kommunikation, Übersetzung und Sinnesveränderung, die die Zukunft von der Art und Weise der heutigen Reproduktion ablösen. Die sexuelle Reproduktion als symbolische Repräsentation sexueller Unterschiede mache, wie es auf ihrer Internetseite heißt, Platz für eine Vielzahl von Formen und Arten der Informationsübertragung, die anstelle von Beziehungen und Verwandtschaft qua Blut einen subtileren Fluss der Vermischung von Zwischenräumen erzeuge. ${ }^{12}$

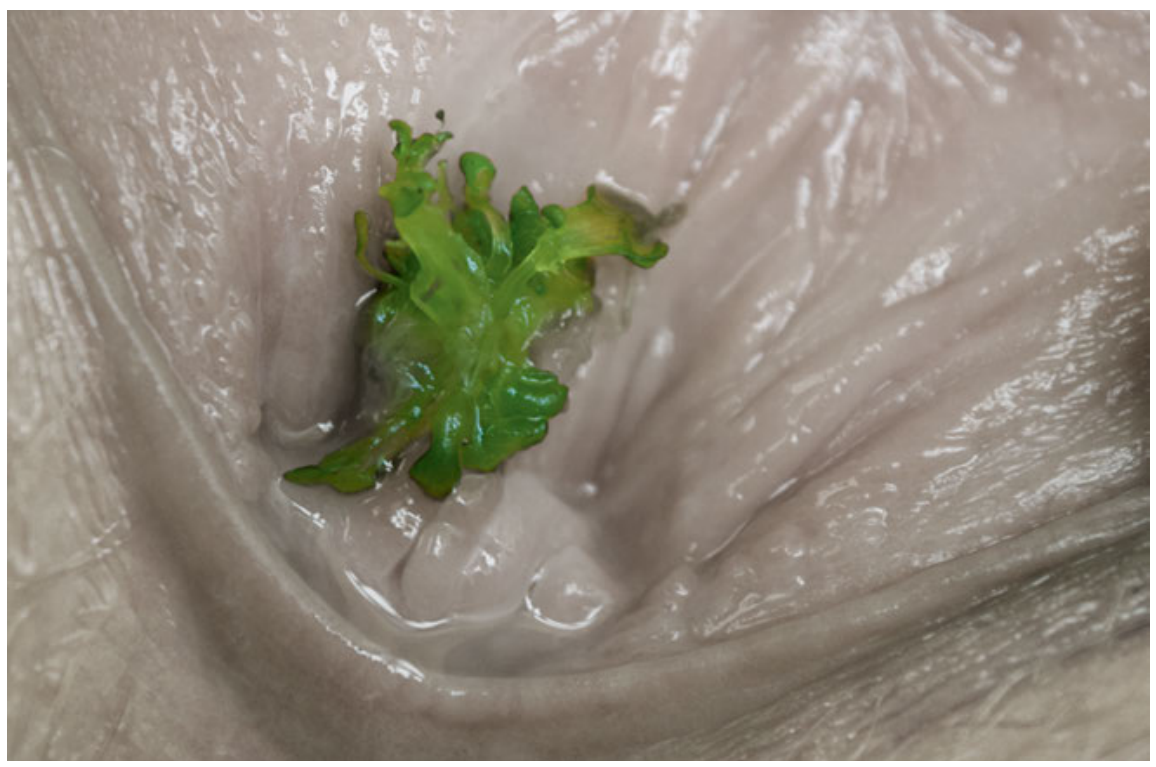

Špela Petrič̌: "Phytoteratology«, 2016, (C) Miha Turšič

\section{Bio-Art und die Reproduktionsweisen des Vegetativen. Herkömmliche Geschlechterkategorisierungen und neue Modelle emergenter Vermischungen}

Špela Petrič und ihre Kunst stehen hier stellvertretend für Bio-Art-Künstler_innen, die den Diskurs um die ökologische und biotechnische Zukunft erweitern wollen, zu einer partizipativen Aneignung neuer Technologien

12 https://www.spelapetric.org/\#/ectogenesisphytoteratology/; mit Bezugnahme auf Nealin 2015; Woldzko 2015. 
beizutragen beabsichtigen und sich mit ihren künstlerischen Arbeiten theoretisch und politisch verorten, z. B. in den Theoriefeldern des Neuen Materialismus, der Objektorientierten Ontologie, des relationalen Feminismus oder auch der Deleuzianischen Philosophie (siehe dazu die genannten Projekte bei z.B. Wenzel/Blohm 2018; Beloff/Berger/Haapoja 2013; Kluszczynski 2012; Mitchell 2010; Hauser 2008; Kac 2007). In dem Theoriefeld, in dem Bio-Art verankert ist, haben sich feministische Philosoph_innen durchaus hervorgetan und in Auseinandersetzung mit Deleuzes und Guattaris Schriften kritisch und kreativ deren Ideen des Werdens, des Unterschiedes und der Dauer weiter entwickelt: Braidotti bietet das Konzept von Zoë, einer generativen Kraft bzw. des Lebens selbst an und diskutiert dessen Verstrickung mit Politik und Ethik (Braidotti 2002); Grosz liest Deleuzes Arbeit durch Bergson und Darwin und konzentriert sich dabei sowohl auf die Öffnung in die Zukunft als auch auf die Beziehung zwischen dem Lebenden und dem Nicht-Lebenden (Grosz 2011). Colebrook plädiert für einen passiven Vitalismus, eine Philosophie, die vom "Vorstellen als solchem" ausgeht (Colebrook 2010, 185) und so den eigenen Standort reflexiv werden lässt. Eine Spurensuche im Feld der Bio-Art zeigt hier viele Verbindungen (vgl. dazu: Radomska 2016).

Darüber hinaus haben feministische Wissenschaftsforscher_innen auf den dynamischen, aggressiven Charakter von Materie, ihre Unbestimmtheit und den primären Charakter von Beziehungen und Prozessen verwiesen. In Anlehnung an die Quantenphysik konzentriert sich Karen Barad auf die Verflechtung der Materie mit dem Diskursiven sowie der von Zeit, Raum und Materie und zeigt, dass nicht individuelle, vorgängig mit bestimmten Eigenschaften versehene Entitäten und Dimensionen, sondern emergente Phänomene die primären ontologischen Einheiten darstellen (Barad 2007). Donna Haraway betont die Verflechtung von natürlichem und kulturellem, organischem und anorganischem Material, Materiellem und Immateriellem sowie die mitkonstitutiven Beziehungen zwischen Menschen und Nichtmenschen ("natureculture«, Haraway 2003). Diese Ansätze zielen auf die Konzeptualisierung von Ontologien, in denen Lebendigkeit als materiell unbestimmt, prozessual unabgeschlossen und als durch vielfältige Verbindungen und Intraaktionen hervorgebracht verstanden wird. Zudem fragen diese Autorinnen nach der Beeinflussung, Beeinträchtigung und Verantwortlichkeit für das, was, wie Barad es formuliert, durch »agentielle Schnitte (Barad 2007, 139) ausgeschlossen ist oder nie materialisiert wird. Damit werfen sie Fragen auf, die bislang nicht gestellt wurden, z.B. die Frage, mit welchen Entitäten man/wir wie leben könnte/n. 
Auf der Suche nach Konzeptionalisierungen von Subjektivität, die nicht auf Menschen beschränkt und auch nicht darauf ausgerichtet sind, Andere(s) zu unterwerfen, spielt die Idee der symmetrischen Ko-Laboration zwischen verschiedenen Arten eine herausgehobene Rolle. Donna Haraway hat im Zuge ihrer Aufforderung, sich auch mit anderen Arten "verwandt« zu machen (Haraway 2016 [2018], 137), eine Vielzahl von Lebewesen und Entitäten entworfen, die als virtuose Opportunist_innen und Arbeiter_innen mit einem großen Maß an kognitivem und affektivem Feintuning zu Gefährt_innen werden bzw. werden könn(t)en. Dabei kommen in ihren Beschreibungen überwiegend uns bekannte Lebewesen, wie Hunde, Tauben, Lemuren und Kraken vor. Špela Petrič schreibt dieses Ansinnen fort und verlagert es nicht nur auf die Mikroebene des Molekularen und für das menschliche Auge Unsichtbaren, sondern bezieht es zudem auf pflanzliche Formen des Lebens, für deren molekulare Struktur die Naturwissenschaften bislang kaum Intellekt nachweisen und keinen Affekt zeigen konnten. Mit dieser künstlerischen Erweiterung des Harawayschen Postulats ist die Frage verbunden, ob und wie Verwandtschaft und Gefährtentum auch mit solchen Arten möglich ist, deren Wahrnehmungsfunktionen bislang nur wenig erschlossen sind. Da aber in der naturwissenschaftlichen Forschung Prozesse von Umwelt bezogenen Veränderungen sowie Arten spezifische Flexibilität und Kontextvariabilität viele Fragen möglicher Interaktion und Ko-Laboration aufwerfen, haben sich, wie das Paradigma des "artistic research" zeigt, Teile der naturwissenschaftlichen Forschung dafür geöffnet, den Raum der konzeptuellen Möglichkeiten figurativ und ästhetisch zu erweitern. Hier knüpft nun die Kunst an Theoriefiguren an, die darauf zielen, Kollektivitäten und Partialitäten gleichermaßen zu denken und Termini wie "zwischenartliche Kollaborationen", "Hybridität», "techno-korporale Prozesse«, "materialdiskursiv« finden, wie beispielweise der Internet-Auftritt des Berliner Art Laboratory vielfältig zeigt, umfängliche Verwendung. ${ }^{13}$

Im Sinne dieser Theorieorientierung sind die Objekte, Produkte und Inszenierungen von »Pflanzlichen Anderen« der Bio-Art-Künstlerin Špela Petrič als Entwürfe von Lebensvollzügen zu verstehen, die verschiedene Wesen miteinander in Beziehung bringen, neue Körper-Erfahrungen und andere Bedeutungen von Leben und Lebendigkeit entstehen lassen und Perspektiven auf neue Phänomene, z. B. Reproduktionsweise des Vegetativen (statt sexueller Reproduktion) eröffnen. Mit Haraway gedacht, kann man sich diese neuen Formen verschiedener Miteinander so vorstellen,

13 http://artlaboratory-berlin.org/html/de-event-40.htm; letzter Aufruf am 04.06.2019. 
dass sie als "Apparate der körperlichen Produktion« wirksam werden, als "materiell-semiotische Erzeugungsknoten" (Haraway 1995 [1988], 96), an denen Biologisches, Technisches, Materielles, Diskursives, Textuelles, Ökonomisches, Künstlerisches und Politisches zusammen kommen. Haraway hatte das Konzept "Apparat der körperlichen Produktion" schon vor längerer Zeit entwickelt, um dem Verständnis wissenschaftlicher Objektivität entgegen zu wirken, das auf der Annahme beruht, das Erkenntnisobjekt sei passiv und stumm. Sie geht vielmehr davon aus, dass Objekte, Körper und Organismen weder epistemologisch noch ontologisch vorgängige Entitäten seien, die darauf warteten, entdeckt zu werden. Sie könnten auch nicht als bloße Endprodukte sozialer Konstruktion verstanden werden. Vielmehr materialisierten sich Körper und deren Bedeutungen durch bestimmte machtvolle Praktiken und würden zum "Apparat körperlicher Produktion", - im Sinne des lateinischen apparare: etwas in Erscheinung bringen, sichtbar machen (Haraway 1995 [1988]). Karen Barad hat die mit dieser Begrifflichkeit verbundene Überlegung aufgegriffen und argumentiert, dass Körper ebenso wie Bedeutungen sich erst durch bestimmte Apparate manifestieren, d.h. erst durch materiell-diskursive Praktiken sichtbar werden, die ein jeweils spezifisches Phänomen konstituieren helfen und zugleich auch Teil desselben sind (Barad 2007, 230).

Solche "Apparate körperlicher Produktion" bilden also zum einen generative Orte, die als konkrete Kontexte zu Gegenständen künstlerischer und philosophischer Untersuchungen werden und als spannungsvolle Verschränkung ein bestimmtes Phänomen in Gang setzen - z. B. mikrobiologische Erzeugung von Zellgewebe zweier Arten oder Techniken zur Befruchtung von Pflanzen. Zum anderen sind sie auch kritische Erzählfiguren, Instrumente technikphilosophischer und bio-künstlerischer Analysen von Narrativen, die Fragen der Verbindung von Macht, Körper und Technik in den Blick nehmen.

In dem Bemühen von Špela Petrič, die Perspektive von Organismen einzunehmen und an einem System Natur teilzunehmen, das sie mit Hilfe von Methoden der Biotechnik bearbeitet, verändert und mit dem sie z.B. pflanzenmenschliche Entitäten - »Pflanzenembryos" - herstellt, zeigt sich ein Blick auf konkrete Apparate, Gefüge, Verschränkungen, die heute Leben, Körper und Geschlecht machen. Im globalen Technobiokapitalismus bestimmt und übersetzt sich das Verhältnis von Körper, Geschlecht und Technik neu und so treten auch neue Formen des gemeinschaftlichen Vollzugs von Leben in Erscheinung, die, so könnte man in Erweiterung von Haraway formulieren, als materiell-semiotische Apparate körperlicher Re-Produktion wirksam werden. Diese werden mit anderen bio- 
medialen Informationsübertragungsmethoden wahrgenommen und sind dementsprechend von anderen Leerstellen (z.B.: ohne geschlechtliche Markierung) und Fragezeichen (z.B.: wohin hat sich die Bedeutung der geschlechtlichen Codierungen verlagert?) begleitet.

Petričs Entwürfe von Reproduktionsweisen, die sexuelle noch geschlechtliche Codierungen in Frage stellen, spiegeln die technische Überformung und Veränderung sexueller Reproduktion und die damit verbundene Relativierung binärer geschlechtlicher Unterschiede samt deren symbolischer Repräsentation. Damit wird die differenzierende Wirkung der Kategorie Geschlecht im Allgemeinen in Frage gestellt. Indem die künstlerischen Projekte von Špela Petrič Reproduktion als artenübergreifend und nicht als sexuell-geschlechtlich inszenieren, wird sichtbar, dass die Kategorie es heute weniger als früher vermag, Relationen zu erzeugen, weil die technische Entgrenzung von Leben wie auch die technologischen Mittel zur Herstellung und Erzeugung von Leben damit begonnen haben, die Beziehungen zwischen sex und gender aufzulösen. Zugleich ist mit der Verbreitung der neuen Lebenstechnologien verbunden, >Arten nicht länger als abgeschlossene Einheiten und als kategoriale Unterscheidung zu verstehen.

Haraway hat aus dieser technisch-technologischen Entwicklung ein politisches Programm abgeleitet, Sie schreibt:

Wenn es jemals artenübergreifende ökologische Gerechtigkeit geben soll, die die Diversität menschlicher Leute einschließt, ist es jetzt höchste Zeit, dass FeministInnen Vorstellungen, Theorien und Aktionen entwerfen, welche die Verbindung von Genealogie und Verwandtschaft sowie von Verwandtschaft und Spezies auflösen (Haraway 2018 [2016], 141).

Um diese transformativen Prozesse - zu denen zentral die De-Zentrierung der Kategorie Geschlecht gehört - gleichermaßen zu begreifen wie auch zu gestalten, sind angemessene Begriffe und ggf. neu zu entwickelnde Modelle erforderlich; "Wissen", so Haraway, "ist auf jeder Ebene seiner Artikulation eine situierte Auseinandersetzung". Die gegenwärtige Situation ist davon bestimmt, dass Bio- und Gentechnologien nicht nur das Verhältnis von sex und gender verändert haben, es »steht die Grenze zwischen Tier und Mensch genauso auf dem Spiel wie die zwischen Maschine und Organismus" (Haraway 1995 [1988], 95). Eine Folge davon sei, so postuliert sowohl Haraway als auch die Kunst von Petrič, dass neue "Intra-Aktionen« zwischen `Arten die differenzierende Wirkung von Geschlecht relativieren. 
Künstlerische Entwürfe wie "Skotopoiesis" und "Phytoteratology" sind Versuche, lebendige Materie als dynamisch und agentiell darzustellen. Sie wollen vor Augen führen, dass Menschen notwendigerweise und konstitutiv als Wesen angesehen werden müssen, die in eine Vielzahl von Beziehungen zu menschlichen Akteur_innen wie auch zu nicht-menschlichen Agenten verwickelt sind. Ziel der Bio-Art von Špela Petrič ist es, den verwischten Grenzen zwischen den Kategorien und den Spezies Ausdruck zu verleihen. Diese Kunst mischt sich ein in die Interaktion zwischen Naturwissenschaft, Theorie und Kunst und wird dort aktiv, wo Affekte und Empfindungen dazu beitragen, Konzepte und Modelle zu kreieren, und wo auch umgekehrt, Konzepte und Modelle mittels Affekten mobilisiert werden. Nicht zufällig steht dabei das Thema Reproduktion im Zentrum, ein Bereich, der bislang zentral durch die Kategorie Geschlecht bestimmt war. Hier bieten Petričs Installationen nun solche künstlerischen Modelle techno-materieller Verschränkungen an, in denen - dies suggeriert die geschlechtskategoriale Leerstelle in Petričs Kunst - die Kategorie Geschlecht als zukünftig bedeutungslos dargestellt wird. Unklar bleibt dabei allerdings, ob die neuen techno-materiellen Verschränkungen tatsächlich dazu in der Lage sind, die differenzierende, binär strukturierende Bedeutung von Geschlecht auszuhebeln und stattdessen symmetrische Beziehungen hervor zu bringen vermögen, die der künstlerische Entwurf als mögliche Zukunft imaginiert. Irritierend bleibt, dass das Verschwinden einer so machtvollen Kategorie wie Geschlecht und der damit verbundenen sozialen Ungleichheiten und gesellschaftspolitischen Bedeutungen in Petričs Kunst als so leise und so vollständig inszeniert wird. Ohne Hinweise auf die Kämpfe und Verluste, von denen solche Veränderungen und Verschiebungen in der Regel begleitet sind, transportieren die künstlerischen Visionen auch ein technikaffirmierendes Moment einer allzu bereitwilligen Mitarbeit der Kunst an den biowissenschaftlichen Forschungen. Wenn aber deren beunruhigende Aspekte und Dimensionen mitsamt der Kategorie Geschlecht zum Verschwinden gebracht werden, verringern sich auch die Möglichkeiten, Macht, Gewalt, Widersprüche und Paradoxien im Themenfeld Reproduktion und Gender zu thematisieren. Für das "Staying with the trouble« (Haraway 2016) hingegen könnte die Beibehaltung bzw. Wiederaufnahme der Kategorie Geschlecht sich noch als hilfreich erweisen. 


\section{Literatur}

Alaimo, Stacy / Hekman, Susan (2008): Material Feminisms. Bloomington/Indianapolis.

Art Laboratory Berlin (2018): Flyer zur Ausstellung "Strange Encounters with Vegetal Others«/Špela Petrič 9/2018. Berlin.

Barad, Karen (2003): Posthumanist Performativity: Toward an Understanding of How Matter Comes to Matter. In: Signs. Journal of Women in Culture and Society 28(3), $801-831$.

Barad, Karen (2007): Meeting the Universe Halfway. Quantum Physics and the Entanglement of Matter and Meaning. Durham.

Beloff, Laura / Berger Erich / Haapoja, Terike(2013): Field_Notes. From Landscape to Laboratory. Helsinki: The Finnish Society of Bioart.

Braidotti, Rosi (2002): Metamorphoses: Towards a Feminist Theory of Becoming. London.

Colebrook, Claire (2010): Deleuze and the Meaning of Life. New York/London.

Eggleson, Kathleen (2014): Transatlantic divergences in citizen science ethics - comparative analysis of the DIYbio code of ethics drafts of 2011. In: NanoEthics 8(2), 187-192.

Grosz, Elizabeth (2011): Becoming Undone: Darwinian Reflections on Life, Politics and Art. Durham / London.

Haarmann, Anke (2019): Artistic Research. Eine epistemologische Ästhetik. Bielefeld.

Haraway, Donna J. (1992). The Promises of Monsters: A Regenerative Politics for Inappropriate/d Others. In: Cultural Studies, edited by Lawrence Grossberg, Cary Nelson and Paula A. Treichler. New York, 295-337.

Haraway, Donna J. (1995 [1985]): Ein Manifest für Cyborgs. Feminismus im Streit mit den Technowissenschaften (übersetzt von Fred Wolf). In: dies.: Die Neuerfindung der Natur. Primaten, Cyborgs und Frauen. Frankfurt a. M., 33-72.

Haraway, Donna J. (1995 [1988]): Situiertes Wissen. Die Wissenschaftsfrage im Feminismus und das Priviled einer partialen Perspektive (übersetzt von Helga Kelle). In: dies.: Neuerfindung der Natur. Primaten, Cyborgs und Frauen. Frankfurt a. M., 73-97.

Haraway, Donna J. (1995 [1989]): Die Biopolitik postmoderner Körper. Konstitutionen des Selbst im Diskurs des Immunsystems (übersetzt von Immanuel Stieß). In: dies.: Neuerfindung der Natur. Primaten, Cyborgs und Frauen. Frankfurt a. M., 160-199.

Haraway Donna J. (1997): Modest_Witness@Second_Millenium. FemaleManC_Meets_ OncoMouse ${ }^{\mathrm{TM}}$. Feminism and Technoscience. London/New York.

Haraway, Donna J. (2003): The Companion Species Manifesto. Dogs, people and Significant Otherness. Chicago.

Haraway, Donna J. (2008). When the Species Meet. Minneapolis/London.

Haraway, Donna J. (2018 [2016]): Unruhig bleiben. Die Verwandtschaft der Arten im Chthuluzän. Frankfurt a. M. / New York.

Hauser, Jens (Hg.) (2008): Sk-interfaces: Exploding Borders - Creating Membrances in Art, Technology and Society. Liverpool.

https://ec.europa.eu/programmes/horizon2020/en/h2020-section/science-and-society. https://www.forschung.tu-berlin.de/servicebereich/menue/forschung_an_der_tu/citizen_science_projekte_2018/mind_the_fungi/.

https://www.hackteria.org/ OPEN SOURCE BIOLOGICAL ART.

https://www.spelapetric.org/\#/ectogenesisphytoteratology/.

Kac, Eduardo(2000): GFP Bunny. http://www.ekac.org/gfpbunny.html\#gfpbunnyanchor. 
Kac, Eduardo, ed.( 2007). Signs of Life: Bio Art and Beyond. Cambridge.

Kluszczynski, Ryszard W. (2012): Crude Life. The Tissue Culture \& Art Project. Oron Catts \& Ionat Zurr. Gdansk.

Lehner, Maximilian (2017): Can Art-Technology Co-Operations Provide a Paradigm for Artistic Research? In: Vila, Irma/Alsina, Pau (Hrsg.): Art and Research. Artnodes. No. 20, 57-66.

Mêtraux, Alexandre/Straub, Jürgen u.a. (Hrsg.) (2016): Prothetische Transformation des Menschen. Ersatz, Ergänzung, Erweiterung. Bochum.

Mitchell, Robert (2010): Bioart and the Vitality of Media. Seattle / London.

Müller- Jung, Joachim (2018): BioArt boomt. Wahrheiten, die uns nur durch Kunst bewusst werden. In: FAZ 26.09.2018.

Myers, William (2015): Bio art. Altered realities. London.

Radomska, Marietta (2016): Uncontainable Life. A biophilosophy of Bioart. Linköping: Linköping Studies in Arts and Science, No. 666.

Rapp, Regine/da Luz, Christian (2018): Trans-, Post- und darüber hinaus. Einige Reflexionen über Maschinen und das Biologische. In: Wenzel, Käthe/Blohm, Manfred (Hrsg.): HalfLife. Maschinen / Organismen - künstlerische Positionen in Zeiten von Klimawandel und Artensterben. Hannover, 14-29.

Reichle, Ingeborg (2005): Kunst aus dem Labor. Zum Verhältnis von Kunst und Wissenschaft im Zeitalter der Technoscience. Wien / New York.

Serres, Michel (2003): Erfindet Euch neu! Eine Liebeserklärung an die vernetzte Generation. Berlin.

Strathern, Marilyn 1992: Reproducing the future. Manchester.

Vohland, Katrin/Göbel, Claudia (2017): Open Science und Citizen Science als symbiotische Beziehung? In: TATuP Zeitschrift für Technikfolgenabschätzung in Theorie und Praxis, Karlsruhe, Jg. 26, Nr. 1-2, 18-24, DOI: https://doi. org/10.14512/ tatup.26.1-2.18.

Wenzel, Käthe/Blohm, Manfred (Hrsg.) (2018): HalfLife. Maschinen/Organismen künstlerische Positionen in Zeiten von Klimawandel und Artensterben. Hannover.

Wolodzko, Agnieszka (2015): Materiality of Affect. How art can reveal the more subtle reality of an encounter. In: Braidotti, Rosi/Dophijn, Rick (Hrsg.): This Deleuzian Century. Art, activism, Life. Amsterdam/New York, 169-184. 